\title{
The Estimates of the Upper Bounds of Hausdorff Dimensions for the Global Attractor for a Class of Nonlinear Coupled Kirchhoff-Type Equations
}

\author{
Guoguang Lin, Ming Zhang \\ Department of Mathematics, Yunnan University, Kunming, China \\ Email: gglin@ynu.edu.cn, 332026295@qq.com
}

How to cite this paper: Lin, G.G. and Zhang, M. (2018) The Estimates of the Upper Bounds of Hausdorff Dimensions for the Global Attractor for a Class of Nonlinear Coupled Kirchhoff-Type Equations. Advances in Pure Mathematics, 8, 1-10. https://doi.org/10.4236/apm.2018.81001

Received: December 7, 2017

Accepted: January 6, 2018

Published: January 9, 2018

Copyright $(9) 2018$ by authors and Scientific Research Publishing Inc. This work is licensed under the Creative Commons Attribution International License (CC BY 4.0).

http://creativecommons.org/licenses/by/4.0/

\begin{abstract}
This paper deals with the Hausdorff dimensions of the global attractor for a class of Kirchhoff-type coupled equations with strong damping and source terms. We obtain a precise estimate of upper bound of Hausdorff dimension of the global attractor.
\end{abstract}

\section{Keywords}

Kirchhoff-Type Equations, The Global Attractor, Hausdorff Dimension

\section{Introduction}

Guohuang Lin, Ming Zhang [1] studied the initial boundary value problem for a class of Kirchhoff-type coupled equations and obtained the existence of the global attractor. Next, in this paper, we consider the Hausdorff dimensions for the global attractor for the following Kirchhoff-type equations:

$$
\begin{gathered}
u_{t t}-M\left(\|\nabla u\|^{2}+\|\nabla v\|^{2}\right) \Delta u-\beta \Delta u_{t}+g_{1}(u, v)=f_{1}(x) \\
v_{t t}-M\left(\|\nabla u\|^{2}+\|\nabla v\|^{2}\right) \Delta v-\beta \Delta v_{t}+g_{2}(u, v)=f_{2}(x) \\
u(x, 0)=u_{0}(x), u_{t}(x, 0)=u_{1}(x), x \in \Omega \\
v(x, 0)=v_{0}(x), v_{t}(x, 0)=v_{1}(x), x \in \Omega \\
\left.u\right|_{\partial \Omega}=\left.v\right|_{\partial \Omega}=0
\end{gathered}
$$

where $\Omega$ is a bounded domain in $R^{2}$ with the smooth boundary $\partial \Omega, \beta>0$ 
is a constant. $M(s)$ is a nonnegative $C^{1}$ function, $-\Delta u_{t}$ and $-\Delta v_{t}$ are strongly damping terms, $g_{1}(u, v)$ and $g_{2}(u, v)$ are nonlinear source terms, $f_{1}(x)$ and $f_{2}(x)$ are given forcing function.

Jingzhu $\mathrm{Wu}$, Guoguang Lin [2] consider a class of damped Bossinesq equation:

$$
\begin{gathered}
u_{t t}+\alpha u_{t}-u_{x x}+u^{2 k+1}=f(x), x \in \Omega, t>0 \\
u(x, 0)=u_{0}(x) \\
u(x, t)=u(x+1, t)
\end{gathered}
$$

where $\Omega \subset R, \alpha>0$, then they obtain the existence of the global attractor and the limited of Hausdorff dimension and the limited of Fractal dimension.

Xiaoming Fan, Shengfan Zhou [3] consider the following non-autonomous strongly damped wave equation of non-degenerate Kirchhoff-type:

$$
\begin{gathered}
u_{t t}-\alpha \Delta u_{t}-\left(\beta+\gamma\left(\int_{\Omega}|\nabla u|^{2} \mathrm{~d} x\right)^{p}\right) \Delta u+h\left(u_{t}\right)+f(u, t)=g(x, t), x \in \Omega, t>\tau \\
\left.u(x, t)\right|_{x \in \partial \Omega}=0, t \geq \tau \\
u(x, \tau)=u_{0 \tau}(x), u_{t}(x, \tau)=u_{1 \tau}(x), x \in \Omega
\end{gathered}
$$

where $u=u(x, t)$ is a real-valued function on $\Omega \times[\tau,+\infty), \tau \in R, \Omega$ is an open bounded set of $R^{n}, n=1,2,3$ with a smooth boundary $\partial \Omega, \alpha>0$ is called the strong damping, $\beta>0, \rho>-1, \gamma \geq 0 . h \in C^{1}(R ; R)$, $f \in C^{1}(R \times R ; R), g(\cdot, t) \in C_{b}\left(R, L^{2}(\Omega)\right), \quad C_{b}\left(R, L^{2}(\Omega)\right)$ is the set of continuous bounded functions from $R$ into $L^{2}(\Omega)$. And then, they obtained a precise estimate of upper bound of Hausdorff dimension of kernel sections, which decreases as the strong damping grows for large strong damping under some conditions, particularly in the autonomous case.

Guoguang Lin, Yunlong Gao [4] concerned the following nonlinear Higher-order Kirchhoff-type equations:

$$
\begin{gathered}
u_{t t}+(-\Delta)^{m} u_{t}+\left(\alpha+\beta\left\|\nabla^{m} u\right\|^{2}\right)^{q}(-\Delta)^{m} u+g(u)=f(x),(x, t) \in \Omega \times[0,+\infty)(1.1 \\
u(x, 0)=u_{0}(x), u_{t}(x, 0)=u_{1}(x), x \in \Omega \\
u(x, t)=0, \frac{\partial^{i} u}{\partial v^{i}}=0, i=1, \cdots, m-1, x \in \partial \Omega, t \in[0,+\infty)
\end{gathered}
$$

where $m>1$ is an integer constant, $\alpha>0, \beta>0$ are constants and $q$ is a real number. $\Omega$ is a bounded domain of $R^{n}$ with a smooth boundary $\partial \Omega$ and $v$ is the unit outward normal on $\partial \Omega . g(u)$ is a nonlinear function specified later. And they obtained the existence of the global attractor. In this case, they considered that the estimation of the upper bounds of Hausdorff for the global attractors is obtained.

\section{Hausdorff Dimensions of the Global Attractor}

In this paper, some inner product, norms, abbreviations and some assumptions 
$\left(\mathrm{H}_{1}\right)-\left(\mathrm{H}_{4}\right)$ and notations needs in the proof of our results in refer to [1].

\subsection{Differentiability of the Semigroup}

In order to estimate dimensions, we suppose:

$\left(\mathrm{H}_{5}\right)$ For every $L>0$, there exist $k=k(L)$, such that:

$$
\begin{aligned}
& \left\|g_{i u}(u, v)-g_{i u}(\gamma u+(1-\gamma) \bar{u}, v)\right\|_{L^{\infty}(\Omega)} \leq k\|\nabla \bar{u}-\nabla u\|^{\delta_{1}}+k\|\nabla \bar{v}-\nabla v\|^{\delta_{1}} \\
& \left\|g_{i v}(u, v)-g_{i v}(u, \gamma v+(1-\gamma) \bar{v})\right\|_{L^{\infty}(\Omega)} \leq k\|\nabla \bar{u}-\nabla u\|^{\delta_{1}}+k\|\nabla \bar{v}-\nabla v\|^{\delta_{1}}
\end{aligned}
$$

where $\quad u, \bar{u}, v, \bar{v} \in H_{0}^{1}(\Omega) ; \quad\|\nabla u\|,\|\nabla \bar{u}\|,\|\nabla v\|,\|\nabla \bar{v}\| \leq L ; \quad \lambda \in(0,1) ; \quad \delta_{1}>0 ;$ $i=1,2$.

$\left(\mathrm{H}_{6}\right)$ There exists constant $\mu_{0}, \mu_{1}, \mu_{2}$, such that

$$
1<\mu_{0} \leq M(s) \leq \mu_{1}, \mu_{2}=\left\{\begin{array}{l}
\mu_{0}, \frac{\mathrm{d}}{\mathrm{d} t}\left(\|\nabla \theta\|^{2}+\|\nabla \omega\|^{2}\right)>0, \\
\mu_{1}, \frac{\mathrm{d}}{\mathrm{d} t}\left(\|\nabla \theta\|^{2}+\|\nabla \omega\|^{2}\right)<0 .
\end{array}\right.
$$

We define $A=-\Delta, \quad E_{0}=H_{0}^{1}(\Omega) \times H_{0}^{1}(\Omega) \times L_{2}(\Omega) \times L_{2}(\Omega)$. The inner product and norm in $E_{0}$ space are defined as follows:

$$
\forall \varphi_{i}=\left(u_{i}, v_{i}, p_{i}, q_{i}\right) \in E_{0},(i=1,2)
$$

we have

$$
\begin{gathered}
\left(\varphi_{1}, \varphi_{2}\right)_{E_{0}}=\left(A^{\frac{1}{2}} u_{1}, A^{\frac{1}{2}} u_{2}\right)+\left(A^{\frac{1}{2}} v_{1}, A^{\frac{1}{2}} v_{2}\right)+\left(p_{1}, p_{2}\right)+\left(q_{1}, q_{2}\right) \\
\left\|\varphi_{1}\right\|_{E_{0}}^{2}=\left(\varphi_{1}, \varphi_{1}\right)_{E_{0}}=\left\|A^{\frac{1}{2}} u_{1}\right\|^{2}+\left\|A^{\frac{1}{2}} v_{1}\right\|^{2}+\left\|p_{1}\right\|^{2}+\left\|q_{1}\right\|^{2}
\end{gathered}
$$

Setting $\forall \varphi=(u, v, p, q)^{\mathrm{T}} \in E_{0}, \quad p=u_{t}+\varepsilon u, \quad q=v_{t}+\varepsilon v$, the Equations (1.1)-(1.5) is equivalent to

$$
\varphi_{t}+H(\varphi)=F(\varphi)
$$

where

$$
\begin{gathered}
H(\varphi)=\left(\begin{array}{c}
\varepsilon u-p \\
\varepsilon v-q \\
-\varepsilon p+\beta A p+\varepsilon^{2} u+(1-\varepsilon \beta) A u \\
-\varepsilon q+\beta A q+\varepsilon^{2} v+(1-\varepsilon \beta) A v
\end{array}\right) \\
F(\varphi)=\left(\begin{array}{c}
0 \\
0 \\
\left(1-M\left(\|\nabla u\|^{2}+\|\nabla v\|^{2}\right)\right) A u-g_{1}(u, v)+f_{1}(x) \\
\left(1-M\left(\|\nabla u\|^{2}+\|\nabla v\|^{2}\right)\right) A v-g_{2}(u, v)+f_{2}(x)
\end{array}\right)
\end{gathered}
$$

Lemma 2.1 For any $\varphi=(u, v, p, q)^{\mathrm{T}} \in E_{0}$, we have 


$$
(H(\varphi), \varphi)_{E_{0}} \geq \frac{\varepsilon}{4}\|\varphi\|_{E_{0}}^{2}+\frac{\beta}{4}\left\|A^{\frac{1}{2}} p\right\|^{2}+\frac{\beta}{4}\left\|A^{\frac{1}{2}} q\right\|^{2}
$$

Proof.

By (2.3), we get

$$
\begin{aligned}
(H(\varphi), \varphi)_{E_{0}}= & \left(A^{\frac{1}{2}}(\varepsilon u-p), A^{\frac{1}{2}} u\right)+\left(A^{\frac{1}{2}}(\varepsilon v-q), A^{\frac{1}{2}} v\right) \\
& +\left(-\varepsilon p+\beta A p+\varepsilon^{2} u+(1-\varepsilon \beta) A u, p\right) \\
& +\left(-\varepsilon q+\beta A q+\varepsilon^{2} v+(1-\varepsilon \beta) A v, q\right) \\
= & \varepsilon\left\|A^{\frac{1}{2}} u\right\|^{2}-\varepsilon\|p\|^{2}+\beta\left\|A^{\frac{1}{2}} p\right\|^{2}+\varepsilon^{2}(u, p)-\varepsilon \beta\left(A^{\frac{1}{2}} u, A^{\frac{1}{2}} p\right) \\
& +\varepsilon\left\|A^{\frac{1}{2}} v\right\|^{2}-\varepsilon\|q\|^{2}+\beta\left\|A^{\frac{1}{2}} q\right\|^{2}+\varepsilon^{2}(v, q)-\varepsilon \beta\left(A^{\frac{1}{2}} v, A^{\frac{1}{2}} q\right)
\end{aligned}
$$

By using holder inequality and Young's inequality and Poincare inequality, we deal with the terms in (2.9) by as follows:

$$
\begin{gathered}
\varepsilon^{2}(u, p) \geq-\frac{\varepsilon^{2}}{2 \lambda_{1}}\left\|A^{\frac{1}{2}} u\right\|^{2}-\frac{\varepsilon^{2}}{2}\|p\|^{2} \\
-\varepsilon \beta\left(A^{\frac{1}{2}} u, A^{\frac{1}{2}} p\right) \geq-\frac{\varepsilon^{2} \beta}{2}\left\|A^{\frac{1}{2}} u\right\|^{2}-\frac{\beta}{2}\left\|A^{\frac{1}{2}} p\right\|^{2} \\
\varepsilon^{2}(v, q) \geq-\frac{\varepsilon^{2}}{2 \lambda_{1}}\left\|A^{\frac{1}{2}} v\right\|^{2}-\frac{\varepsilon^{2}}{2}\|q\|^{2} \\
-\varepsilon \beta\left(A^{\frac{1}{2}} v, A^{\frac{1}{2}} q\right) \geq-\frac{\varepsilon^{2} \beta}{2}\left\|A^{\frac{1}{2}} v\right\|^{2}-\frac{\beta}{2}\left\|A^{\frac{1}{2}} q\right\|^{2}
\end{gathered}
$$

By $0<\varepsilon<\min \left\{\frac{3}{4} \cdot \frac{2 \lambda_{1}}{1+\beta \lambda_{1}}, \frac{-5+\sqrt{25+8 \lambda_{1} \beta}}{4}\right\}$ and substituting (2.11)-(2.14) into (2.10), we obtain

$$
\begin{aligned}
(H(\varphi), \varphi)_{E_{0}} \geq & \left(\varepsilon-\frac{\varepsilon^{2}}{2 \lambda_{1}}-\frac{\varepsilon^{2} \beta}{2}\right)\left(\left\|A^{\frac{1}{2}} u\right\|^{2}+\left\|A^{\frac{1}{2}} v\right\|^{2}\right) \\
& \left.+\left(-\varepsilon-\frac{\varepsilon^{2}}{2}\right)\left(\|p\|^{2}+\|q\|^{2}\right)+\frac{\beta}{2}\left\|A^{\frac{1}{2}} p\right\|^{2}+\left\|A^{\frac{1}{2}} q\right\|^{2}\right) \\
\geq & \left(\varepsilon-\frac{\varepsilon^{2}}{2 \lambda_{1}}-\frac{\varepsilon^{2} \beta}{2}\right)\left(\left\|A^{\frac{1}{2}} u\right\|^{2}+\left\|A^{\frac{1}{2}} v\right\|^{2}\right)+\left(-\varepsilon-\frac{\varepsilon^{2}}{2}\right)\left(\|p\|^{2}+\|q\|^{2}\right) \\
& \left.+\frac{\beta}{4}\left(\left\|A^{\frac{1}{2}} p\right\|^{2}+\left\|A^{\frac{1}{2}} q\right\|^{2}\right)+\frac{\beta}{4}\left\|A^{\frac{1}{2}} p\right\|^{2}+\left\|A^{\frac{1}{2}} q\right\|^{2}\right)
\end{aligned}
$$




$$
\begin{aligned}
\geq & \left(\varepsilon-\frac{\varepsilon^{2}}{2 \lambda_{1}}-\frac{\varepsilon^{2} \beta}{2}\right)\left(\left\|A^{\frac{1}{2}} u\right\|^{2}+\left\|A^{\frac{1}{2}} v\right\|^{2}\right) \\
& +\left(\frac{\lambda_{1} \beta}{4}-\varepsilon-\frac{\varepsilon^{2}}{2}\right)\left(\|p\|^{2}+\|q\|^{2}\right)+\frac{\beta}{4}\left(\left\|A^{\frac{1}{2}} p\right\|^{2}+\left\|A^{\frac{1}{2}} q\right\|^{2}\right) \\
\geq & \frac{\varepsilon}{4}\left(\left\|A^{\frac{1}{2}} u\right\|^{2}+\left\|A^{\frac{1}{2}} v\right\|^{2}+\|p\|^{2}+\|q\|^{2}\right)+\frac{\beta}{4}\left(\left\|A^{\frac{1}{2}} p\right\|^{2}+\left\|A^{\frac{1}{2}} q\right\|^{2}\right) .
\end{aligned}
$$

Proof finished.

The linearized equations of (1.1)-(1.5), the above equations as follows:

$$
\begin{gathered}
U_{t t}+M^{\prime}\left(\|\nabla u\|^{2}+\|\nabla v\|^{2}\right) 2[(\nabla u, \nabla U)+(\nabla v, \nabla V)] A u \\
+M\left(\|\nabla u\|^{2}+\|\nabla v\|^{2}\right) A U+\beta A U_{t}+g_{1 u}(u, v) U+g_{1 v}(u, v) V=0, \\
V_{t t}+M^{\prime}\left(\|\nabla u\|^{2}+\|\nabla v\|^{2}\right) 2[(\nabla u, \nabla U)+(\nabla v, \nabla V)] A v \\
+M\left(\|\nabla u\|^{2}+\|\nabla v\|^{2}\right) A V+\beta A V_{t}+g_{2 u}(u, v) U+g_{2 v}(u, v) V=0, \\
\left.U(x, 0)\right|_{x \in \partial \Omega}=\left.V(x, 0)\right|_{x \in \partial \Omega}=0, t>0 \\
U(x, 0)=\xi_{1}, U_{t}(x, 0)=\xi_{2} \\
V(x, 0)=\zeta_{1}, V_{t}(x, 0)=\zeta_{2}
\end{gathered}
$$

where $\left(\xi_{1}, \zeta_{1}, \xi_{2}, \zeta_{2}\right) \in E_{0}, \quad\left(u, v, u_{t}, v_{t}\right)=S(t)\left(u_{0}, v_{0}, u_{1}, v_{1}\right)$ is the solution of with $\left(u_{0}, v_{0}, u_{1}, v_{1}\right) \in \mathrm{A}$

Given $\left(u_{0}, v_{0}, u_{1}, v_{1}\right) \in \mathrm{A}$ and $S(t): E_{0} \rightarrow E_{0}$, the solution $S(t)\left(u_{0}, v_{0}, u_{1}, v_{1}\right) \in E_{0}$, by stand methods we can show that for any $\left(\xi_{1}, \zeta_{1}, \xi_{2}, \zeta_{2}\right) \in E_{0}$, the linear initial boundary value problem (2.16)-(2.20) possess a unique solution $\left(U(t), V(t), U_{t}(t), V_{t}(t)\right) \in L^{\infty}\left(0,+\infty ; E_{0}\right)$.

Theorem 2.1 For any $t>0, R>0$, the mapping $S(t): E_{0} \rightarrow E_{0}$ is Frechet differentiable on. Its differential at $\varphi=\left(u_{0}, v_{0}, u_{1}, v_{1}\right)^{\mathrm{T}}$ is the linear operator on

$$
E:\left(\xi_{1}, \zeta_{1}, \xi_{2}, \zeta_{2}\right)^{\mathrm{T}} \rightarrow(U(t), V(t), P(t), Q(t))^{\mathrm{T}}
$$

where $U(t), V(t)$ is the solution of (2.16)-(2.20).

Proof.

Let $\varphi_{0}=\left(u_{0}, v_{0}, u_{1}, v_{1}\right)^{\mathrm{T}} \in E_{0}, \overline{\varphi_{0}}=\left(u_{0}+\xi_{1}, v_{0}+\zeta_{1}, u_{1}+\xi_{2}, v_{1}+\zeta_{2}\right)^{\mathrm{T}} \in E_{0} \quad$ with $\left\|\varphi_{0}\right\|_{E_{0}} \leq R,\left\|\overline{\varphi_{0}}\right\|_{E_{0}} \leq R$, we denote $\left(u, u_{t}\right)^{\mathrm{T}}=S(t) \varphi_{0},\left(\bar{u}, \overline{u_{t}}\right)^{\mathrm{T}}=S(t) \overline{\varphi_{0}} \cdot \mathrm{We}$ can get the Lipchitz property of $S(t)$ on the bounded sets of $E_{0}$, that is

$$
\left\|S(t) \varphi_{0}-S(t) \overline{\varphi_{0}}\right\|_{E_{0}}^{2} \leq \mathrm{e}^{C_{1} t}\left\|\left(\xi_{1}, \zeta_{1}, \xi_{2}, \zeta_{2}\right)\right\|_{E_{0}}^{2}
$$

Let $\theta=\bar{u}-u-U, \omega=\bar{v}-v-V$ is the solution of problem

$$
\begin{aligned}
& \theta_{t t}+M\left(\|\nabla u\|^{2}+\|\nabla v\|^{2}\right) A \theta+\beta A \theta_{t}=h_{1} \\
& \omega_{t t}+M\left(\|\nabla u\|^{2}+\|\nabla v\|^{2}\right) A \omega+\beta A \omega_{t}=h_{2}
\end{aligned}
$$




$$
\begin{gathered}
\theta(0)=\theta_{t}(0)=0 \\
\omega(0)=\omega_{t}(0)=0
\end{gathered}
$$

where

$$
\begin{aligned}
h_{1}= & {\left[M\left(\|\nabla u\|^{2}+\|\nabla v\|^{2}\right)-M\left(\|\nabla \bar{u}\|^{2}+\|\nabla \bar{v}\|^{2}\right)\right] A \bar{u} } \\
& +2 M^{\prime}\left(\|\nabla u\|^{2}+\|\nabla v\|^{2}\right)[(\nabla u, \nabla U)+(\nabla v, \nabla V)] A u \\
& -g_{1}(\bar{u}, \bar{v})+g_{1}(u, v)+g_{1 u}(u, v) U+g_{1 v}(u, v) V, \\
h_{2}= & {\left[M\left(\|\nabla u\|^{2}+\|\nabla v\|^{2}\right)-M\left(\|\nabla \bar{u}\|^{2}+\|\nabla \bar{v}\|^{2}\right)\right] A \bar{v} } \\
& +2 M^{\prime}\left(\|\nabla u\|^{2}+\|\nabla v\|^{2}\right)[(\nabla u, \nabla U)+(\nabla v, \nabla V)] A v \\
& -g_{2}(\bar{u}, \bar{v})+g_{2}(u, v)+g_{2 u}(u, v) U+g_{2 v}(u, v) V .
\end{aligned}
$$

Let $s=\|\nabla u\|^{2}+\|\nabla v\|^{2}, \quad s_{1}=\|\nabla \bar{u}\|^{2}+\|\nabla \bar{v}\|^{2}$, so we can get

$$
\begin{aligned}
& {\left[M(s)-M\left(s_{1}\right)\right] A \bar{u}+2 M^{\prime}\left(\|\nabla u\|^{2}+\|\nabla v\|^{2}\right)[(\nabla u, \nabla U)+(\nabla v, \nabla V)] A u } \\
= & M^{\prime \prime}(\xi)(-\gamma)[(\nabla \bar{u}+\nabla u, \nabla \bar{u}-\nabla u)+(\nabla \bar{v}+\nabla v, \nabla \bar{v}-\nabla v)]^{2} A \bar{u} \\
& -M^{\prime}(s)[(\nabla \bar{u}+\nabla u, \nabla \bar{u}-\nabla u)+(\nabla \bar{v}+\nabla v, \nabla \bar{v}-\nabla v)] A(\bar{u}-u) \\
& -M^{\prime}(s)[(\nabla \bar{u}-\nabla u, \nabla \bar{u}-\nabla u)+(\nabla \bar{v}-\nabla v, \nabla \bar{v}-\nabla v)] A u \\
& -2 M^{\prime}(s)[(\nabla u, \nabla \theta)+(\nabla v, \nabla \omega)] A u
\end{aligned}
$$

And

$$
\begin{aligned}
& g_{1}(u, v)-g_{1}(\bar{u}, \bar{v})+g_{1 u}(u, v) U+g_{1 v}(u, v) V \\
& =g_{1}(u, v)-g_{1}(\bar{u}, v)+g_{1 u}(u, v)(\bar{u}-u)-g_{1 u}(u, v) \theta \\
& \quad+g_{1}(\bar{u}, v)-g_{1}(\bar{u}, \bar{v})+g_{1 v}(u, v)(\bar{v}-v)-g_{1 v}(u, v) \omega .
\end{aligned}
$$

Then, we have

$$
\begin{gathered}
\left(M^{\prime \prime}\left(s_{2}\right)(-\gamma)[(\nabla \bar{u}+\nabla u, \nabla \bar{u}-\nabla u)+(\nabla \bar{v}+\nabla v, \nabla \bar{v}-\nabla v)]^{2} A \bar{u}, \theta_{t}\right) \\
\leq C_{1}\left(\|\nabla \bar{u}-\nabla u\|^{2}+\|\nabla \bar{v}-\nabla v\|^{2}\right)\left\|A^{\frac{1}{2}} \theta_{t}\right\|, \\
\left(-M^{\prime}\left(\|\nabla u\|^{2}+\|\nabla v\|^{2}\right)[(\nabla \bar{u}-\nabla u, \nabla \bar{u}+\nabla u)+(\nabla \bar{v}-\nabla v, \nabla \bar{v}+\nabla v)] A(\bar{u}-u), \theta_{t}\right) \\
\leq C_{2}\left(\|\nabla \bar{u}-\nabla u\|^{2}+\|\nabla \bar{v}-\nabla v\|^{2}\right) \cdot\left\|A^{\frac{1}{2}} \theta_{t}\right\|, \\
\left(-M^{\prime}\left(\|\nabla u\|^{2}+\|\nabla v\|^{2}\right)[(\nabla \bar{u}-\nabla u, \nabla \bar{u}-\nabla u)+(\nabla \bar{v}-\nabla v, \nabla \bar{v}-\nabla v)] A u, \theta_{t}\right) \\
\leq C_{3}\left(\|\nabla \bar{u}-\nabla u\|^{2}+\|\nabla \bar{v}-\nabla v\|^{2}\right)\left\|A^{\frac{1}{2}} \theta_{t}\right\|, \\
\left(-M^{\prime}\left(\|\nabla u\|^{2}+\|\nabla v\|^{2}\right)[(\nabla u, \nabla \theta)+(\nabla v, \nabla \omega)] A u, \theta_{t}\right) \\
\leq C_{4}(\|\nabla \theta\|+\|\nabla \omega\|)\left\|A^{\frac{1}{2}} \theta_{t}\right\| .
\end{gathered}
$$


By using (2.30)-(2.33), we have

$$
\begin{aligned}
& \left(\left[M(s)-M\left(s_{1}\right)\right] A \bar{u}+2 M^{\prime}(s)[(\nabla u, \nabla U)+(\nabla v, \nabla V)] A u, \theta_{t}\right) \\
& \leq C_{5}\left(\|\nabla \bar{u}-\nabla u\|^{2}+\|\nabla \bar{v}-\nabla v\|^{2}\right)\left\|A^{\frac{1}{2}} \theta_{t}\right\|+C_{4}(\|\nabla \theta\|+\|\nabla \omega\|)\left\|A^{\frac{1}{2}} \theta_{t}\right\| .
\end{aligned}
$$

Similarly

$$
\begin{aligned}
& \left(\left[M(s)-M\left(s_{1}\right)\right] A \bar{v}+2 M^{\prime}(s)[(\nabla u, \nabla U)+(\nabla v, \nabla V)] A v, \omega_{t}\right) \\
& \leq C_{6}\left(\|\nabla \bar{u}-\nabla u\|^{2}+\|\nabla \bar{v}-\nabla v\|^{2}\right)\left\|A^{\frac{1}{2}} \omega_{t}\right\|+C_{7}(\|\nabla \theta\|+\|\nabla \omega\|)\left\|A^{\frac{1}{2}} \omega_{t}\right\| .
\end{aligned}
$$

And by using $\left(\mathrm{H}_{5}\right)$

$$
\begin{aligned}
& \left(g_{1}(u, v)-g_{1}(\bar{u}, v)+g_{1 u}(u, v)(\bar{u}-u)-g_{1 u}(u, v) \theta, \theta_{t}\right) \\
& +\left(g_{1}(\bar{u}, v)-g_{1}(\bar{u}, \bar{v})+g_{1 v}(u, v)(\bar{v}-v)-g_{1 v}(u, v) \omega, \theta_{t}\right) \\
& \leq C_{8}\left(\|\nabla \bar{u}-\nabla u\|^{\delta_{1}+1}+\|\nabla \bar{v}-\nabla v\|^{\delta_{1}}\|\nabla \bar{u}-\nabla u\|\right) \cdot\left\|\theta_{t}\right\|+C_{10}\left(\|\theta\| \cdot\left\|\theta_{t}\right\|+\|\omega\| \cdot\left\|\theta_{t}\right\|\right) \\
& +C_{9}\left(\|\nabla \bar{u}-\nabla u\|^{\delta_{1}}\|\nabla \bar{v}-\nabla v\|+\|\nabla \bar{v}-\nabla v\|^{\delta_{1}+1}\right) \cdot\left\|\theta_{t}\right\| \\
& \leq C_{10}\left(\|\theta\| \cdot\left\|\theta_{t}\right\|+\|\omega\| \cdot\left\|\theta_{t}\right\|\right)+C_{11}\left(\|\nabla \bar{u}-\nabla u\|^{\delta_{1}+1}+\|\nabla \bar{v}-\nabla v\|^{\delta_{1}+1}\right) \cdot\left\|\theta_{t}\right\| .
\end{aligned}
$$

Similar

$$
\begin{aligned}
& \left(\left(g_{2 u}(u, v)-g_{2 u}(x, v)\right)(\bar{u}-u)-g_{2 u}(u, v) \theta, \omega_{t}\right) \\
& +\left(\left(g_{2 v}(u, v)-g_{2 v}(\bar{u}, y)\right)(\bar{v}-v)-g_{2 v}(u, v) \omega, \omega_{t}\right) \\
& \leq C_{12}\left(\|\nabla \bar{u}-\nabla u\|^{\delta_{1}+1}+\|\nabla \bar{v}-\nabla v\|^{\delta_{1}+1}\right) \cdot\left\|\omega_{t}\right\|+C_{13}\left(\|\theta\| \cdot\left\|\omega_{t}\right\|+\|\omega\| \cdot\left\|\omega_{t}\right\|\right) .
\end{aligned}
$$

So, we can get

$$
\begin{aligned}
& \frac{\mathrm{d}}{\mathrm{d} t}\left[\left\|\theta_{t}\right\|^{2}+\left\|\omega_{t}\right\|^{2}+\mu_{2}\left(\|\nabla \theta\|^{2}+\|\nabla \omega\|^{2}\right)\right] \\
& \leq C_{15}\left(\|\nabla \bar{u}-\nabla u\|^{2 \delta_{1}+2}+\|\nabla \bar{v}-\nabla v\|^{2 \delta_{1}+2}+\|\nabla \bar{u}-\nabla u\|^{4}+\|\nabla \bar{v}-\nabla v\|^{4}\right) \\
& +C_{14}\left(\left\|\theta_{t}\right\|^{2}+\left\|\omega_{t}\right\|^{2}+\mu_{2}\left(\|\nabla \theta\|^{2}+\|\nabla \omega\|^{2}\right)\right) .
\end{aligned}
$$

Then, by using Gronwall's inequality and (2.21), we obtain

$$
\begin{aligned}
& \left\|\theta_{t}\right\|^{2}+\left\|\omega_{t}\right\|^{2}+\beta_{3}\left(\|\nabla \theta\|^{2}+\|\nabla \omega\|^{2}\right) \\
& \leq C_{15} \mathrm{e}^{C_{14^{t}}} \int_{0}^{t}\|\nabla \bar{u}-\nabla u\|^{2 \delta_{1}+2}+\|\nabla \bar{v}-\nabla v\|^{2 \delta_{1}+2}+\|\nabla \bar{u}-\nabla u\|^{4}+\|\nabla \bar{v}-\nabla v\|^{4} \mathrm{~d} t \\
& \leq C_{17} \mathrm{e}^{C_{16} t}\left(\left\|\left(\xi_{1}, \zeta_{1}, \xi_{2}, \zeta_{2}\right)\right\|_{E_{0}}^{4}+\left\|\left(\xi_{1}, \zeta_{1}, \xi_{2}, \zeta_{2}\right)\right\|_{E_{0}}^{2 \delta_{1}+2}\right) .
\end{aligned}
$$

Then, we get

$$
\begin{aligned}
& \frac{\|\bar{\varphi}(t)-\varphi(t)-U(t)\|_{E_{0}}^{2}}{\left\|\left(\xi_{1}, \zeta_{1}, \xi_{2}, \zeta_{2}\right)\right\|_{E_{0}}^{2}} \leq C_{17} \mathrm{e}^{C_{16} t}\left(\left\|\left(\xi_{1}, \zeta_{1}, \xi_{2}, \zeta_{2}\right)\right\|_{E_{0}}^{2}+\left\|\left(\xi_{1}, \zeta_{1}, \xi_{2}, \zeta_{2}\right)\right\|_{E_{0}}^{2 \delta_{1}}\right) \rightarrow 0(2.40) \\
& \text { as }\left(\xi_{1}, \zeta_{1}, \xi_{2}, \zeta_{2}\right)^{\mathrm{T}} \rightarrow 0 \text { in } E_{0} \text {. The proof is competed. }
\end{aligned}
$$




\subsection{The Upper Bounds of Hausdorff Dimensions for the Global Attractor}

Consider the first variation of (2.6) with initial condition:

$$
\psi_{t}^{\prime}+P(\varphi) \psi=\Gamma_{1}(\varphi) \psi+\Gamma_{2}(\varphi) \psi, \psi(0)=\left(\xi_{1}, \zeta_{1}, \xi_{2}, \zeta_{2}\right)^{\mathrm{T}} \in E_{0}, t>0
$$

where $\psi=(U, V, P, Q)^{\mathrm{T}} \in E_{0}, \quad P=U_{t}+\varepsilon U, Q=V_{t}+\varepsilon V$ and $\varphi=(u, v, p, q)^{\mathrm{T}} \in E_{0}$ is a solution of (2.41),

$$
\begin{gathered}
P(\varphi)=\left(\begin{array}{cccc}
\varepsilon I & 0 & -I & 0 \\
0 & \varepsilon I & 0 & -I \\
\varepsilon^{2} I-(1-\varepsilon \beta) \Delta & 0 & -\varepsilon I-\beta \Delta & 0 \\
0 & \varepsilon^{2} I-(1-\varepsilon \beta) \Delta & 0 & -\varepsilon I-\beta \Delta
\end{array}\right) \\
\Gamma_{1}(\varphi)=\left(\begin{array}{cccc}
0 & 0 & 0 & 0 \\
0 & 0 & 0 & 0 \\
-g_{1 u}(u, v) & -g_{1 v}(u, v) & 0 & 0 \\
-g_{2 u}(u, v) & -g_{2 v}(u, v) & 0 & 0
\end{array}\right) \\
\Gamma_{2}(\varphi) \quad\left(\begin{array}{c}
0 \\
{\left[M\left(\|\nabla u\|^{2}+\|\nabla v\|^{2}\right)-1\right] \Delta u+M^{\prime}\left(\|\nabla u\|^{2}+\|\nabla v\|^{2}\right) 2[(\nabla u, \nabla U)+(\nabla v, \nabla V)] \Delta u} \\
{\left[M\left(\|\nabla u\|^{2}+\|\nabla v\|^{2}\right)-1\right] \Delta v+M^{\prime}\left(\|\nabla u\|^{2}+\|\nabla v\|^{2}\right) 2[(\nabla u, \nabla U)+(\nabla v, \nabla V)] \Delta v}
\end{array}\right)
\end{gathered}
$$

It is easy to show form Theorem 2.1 that (2.41) is a well-posed problem in $E_{0}$, the mapping $S_{\varepsilon}(\tau):\left\{u_{0}, v_{0}, p_{1}=u_{1}+\varepsilon u_{0}, q_{1}=v_{1}+\varepsilon v_{0}\right\} \rightarrow$ $\left\{u(\tau), v(\tau), p(\tau)=u_{t}(\tau)+\varepsilon u(\tau), q(\tau)=v_{t}(\tau)+\varepsilon v(\tau)\right\}$ is frechet differentiable on $E_{0}$ for any $t \geq 0$, its differential at $\varphi=\left(u_{0}, v_{0}, p_{1}, q_{1}\right)^{\mathrm{T}}$ is the linear operator on

$$
E_{0}:\left(\xi_{1}, \zeta_{1}, \xi_{2}, \zeta_{2}\right) \rightarrow(U(t), V(t), P(t), Q(t))^{\mathrm{T}}
$$

where $(U(t), V(t), P(t), Q(t))^{\mathrm{T}}$ is the solution of (2.40).

Lemma 2.2 [5] For any orthonormal family of elements of $\left(E_{0},\|\|_{E_{0}}\right)$, $\left(\xi_{1 j}, \zeta_{1 j}, \xi_{2 j}, \zeta_{2 j}\right)^{\mathrm{T}}, \quad j=1,2, \cdots, n_{1}$, we have

$$
\sum_{j=1}^{n_{1}}\left(\left\|\nabla^{v} \xi_{1 j}\right\|^{2}+\left\|\nabla^{v} \zeta_{1 j}\right\|^{2}\right) \leq 2 \sum_{j=1}^{n_{1}}\left(\lambda_{j}^{v-1}+\lambda_{j}^{\nu-1}\right), v \in[0,1)
$$

Proof. This is a direct consequence of Lemma VI. 6.3 of [5].

Theorem 2.2 If $\left(\mathrm{H}_{1}\right)-\left(\mathrm{H}_{6}\right)$ hold, satisfying, then there exists $\beta>1$, such that the Hausdorff dimension of global attractor $\mathrm{A}$ in $E_{0}$ satisfies

$$
d_{H}(\mathrm{~A}) \leq \min \left\{n_{1} \mid n_{1} \in N, \frac{1}{n_{1}} \sum_{j=1}^{n_{1}}\left(\lambda_{j}^{-1}+\lambda_{j}^{-1}\right)<\frac{\varepsilon}{16 C_{24}}\right\}
$$

where $R_{0}$ is as in Lemma 2.6 in [1].

Proof. Let $n_{1} \in N$ be fixed. Consider $m_{1}$ solutions $\psi_{1}, \psi_{2}, \cdots, \psi_{n_{1}}$ of (2.41). 
At a given time $\tau$, let $B_{n_{1}}(\tau)$ denote the orthogonal projection in $E_{0}$ onto span $\left\{\psi_{1}(s), \psi_{2}(s), \cdots, \psi_{n_{1}}(s)\right\}$.

Let $y_{j}(s)=\left(\xi_{j}, \zeta_{j}, \xi_{j}, \zeta_{j}\right)^{\mathrm{T}} \in E_{0}, \quad j=1,2, \cdots, n_{1}$, be an orthonormal basis of

$$
B_{n_{1}}(s) E_{0}=\operatorname{span}\left\{\psi_{1}(s), \psi_{2}(s), \cdots, \psi_{n_{1}}(s)\right\}
$$

with respect to the inner product $(\cdot, \cdot)_{E_{0}}$ and norm $\|\cdot\|_{E_{0}}$.

Suppose

$$
\varphi(\tau)=(u(\tau), v(\tau), p(\tau), q(\tau))^{\mathrm{T}} \in \mathrm{A}
$$

then $\|\varphi(\tau)\|_{E_{0}} \leq M_{0}, \forall s>\tau$. By $\left\|y_{j}\right\|_{E_{0}}=1$ and Lemma 2.1, we have

$$
\begin{gathered}
-\left(P(\varphi(s)) y_{j}(s), y_{j}(s)\right)_{E_{0}} \leq-\frac{\varepsilon}{4}-\frac{\beta}{4}\left\|\nabla \xi_{2_{j}}\right\|^{2}-\frac{\beta}{4}\left\|\nabla \zeta_{2_{j}}\right\|^{2} \\
\left(\Gamma_{1}(\varphi(s)) y_{j}(s), y_{j}(s)\right)_{E_{0}} \\
\leq C_{18}\left\|\nabla^{-1} \xi_{1 j}\right\| \cdot\left\|\nabla \xi_{2 j}\right\|+C_{19}\left\|\nabla^{-1} \zeta_{1 j}\right\| \cdot\left\|\nabla \xi_{2 j}\right\| \\
\quad+C_{20}\left\|\nabla^{-1} \xi_{1 j}\right\| \cdot\left\|\nabla \zeta_{2 j}\right\|+C_{21}\left\|\nabla^{-1} \zeta_{1 j}\right\| \cdot\left\|\nabla \zeta_{2 j}\right\| .
\end{gathered}
$$

Then, by the Sobolev embedding theorem:

$$
H_{0}^{1}(\Omega) \subset H^{1}(\Omega) \subset H^{-1}(\Omega)
$$

Therefore

$$
\begin{aligned}
& \left(\Gamma_{1}(\varphi(s)) y_{j}(s), y_{j}(s)\right)_{E_{0}} \\
& \leq C_{22}\left\|\xi_{1 j}\right\| \cdot\left\|\nabla \xi_{2 j}\right\|+C_{23}\left\|\zeta_{1 j}\right\| \cdot\left\|\nabla \xi_{2 j}\right\| \\
& +C_{24}\left\|\xi_{1 j}\right\| \cdot\left\|\nabla \zeta_{2 j}\right\|+C_{25}\left\|\zeta_{1 j}\right\| \cdot\left\|\nabla \zeta_{2 j}\right\| \\
& \leq C_{26}\left(\left\|\xi_{1 j}\right\|^{2}+\left\|\zeta_{1 j}\right\|^{2}\right)+\frac{\beta}{8}\left\|\nabla \xi_{2 j}\right\|^{2}+\frac{\beta}{8}\left\|\nabla \zeta_{2 j}\right\|^{2} .
\end{aligned}
$$

By Young's inequality, we have

$$
\begin{aligned}
& \left(\Gamma_{2}(\varphi(s)) y_{j}(s), y_{j}(s)\right)_{E_{0}} \\
& \leq\left(1-\mu_{0}\right)\left\|\nabla \xi_{1 j}\right\| \cdot\left\|\nabla \xi_{2 j}\right\|+2 R_{0}^{2} k_{0}\left\|\nabla \xi_{1 j}\right\| \cdot\left\|\nabla \xi_{2 j}\right\|+2 R_{0}^{2} k_{0}\left\|\nabla \zeta_{1 j}\right\| \cdot\left\|\nabla \xi_{2 j}\right\| \\
& +\left(1-\mu_{0}\right)\left\|\nabla \zeta_{1 j}\right\| \cdot\left\|\nabla \zeta_{2 j}\right\|+2 R_{0}^{2} k_{0}\left\|\nabla \xi_{1 j}\right\| \cdot\left\|\nabla \zeta_{2 j}\right\|+2 R_{0}^{2} k_{0}\left\|\nabla \zeta_{1 j}\right\| \cdot\left\|\nabla \zeta_{2 j}\right\| \\
& \leq \frac{\lambda_{j}^{\frac{1}{2}}\left(1-\mu_{0}\right)}{2}+2 \lambda_{j}^{\frac{1}{2}} R_{0}^{2} k_{0}+\frac{\lambda_{j}^{\frac{1}{2}}\left(1-\mu_{0}\right)}{2}+2 \lambda_{j}^{\frac{1}{2}} R_{0}^{2} k_{0} .
\end{aligned}
$$

So exist $\beta$ satisfying

$$
\begin{aligned}
& -\frac{\beta}{8}\left\|\nabla \xi_{2 j}\right\|^{2}+\frac{\lambda_{j}^{\frac{1}{2}}\left(1-\mu_{0}\right)}{2}+2 \lambda_{j}^{\frac{1}{2}} R_{0}^{2} k_{0} \leq \frac{\varepsilon}{16} \\
& -\frac{\beta}{8}\left\|\nabla \zeta_{2 j}\right\|^{2}+\frac{\lambda_{j}^{\frac{1}{2}}\left(1-\mu_{0}\right)}{2}+2 \lambda_{j}^{\frac{1}{2}} R_{0}^{2} k_{0} \leq \frac{\varepsilon}{16}
\end{aligned}
$$

We obtain 


$$
\begin{aligned}
& p_{n_{1}}(s)= \sum_{j=1}^{n_{1}}\left(-P(\varphi(s))+\Gamma_{1}(\varphi(s))+\Gamma_{2}(\varphi(s)) y_{j}(s), y_{j}(s)\right)_{E_{0}} \\
& \leq \sum_{j=1}^{n_{1}}\left(-\frac{\varepsilon}{4}-\frac{\beta}{4}\left\|\nabla \xi_{2 j}\right\|^{2}-\frac{\beta}{4}\left\|\nabla \zeta_{2 j}\right\|^{2}+\frac{\beta}{8}\left\|\nabla \xi_{2 j}\right\|^{2}+\frac{\beta}{8}\left\|\nabla \zeta_{2 j}\right\|^{2}\right) \\
&+\sum_{j=1}^{n_{1}}\left(\frac{\lambda_{j}^{\frac{1}{2}}\left(1-\mu_{0}\right)}{2}+2 \lambda_{j}^{\frac{1}{2}} R_{0}^{2} k_{0}+\frac{\lambda_{j}^{\frac{1}{2}}\left(1-\mu_{0}\right)}{2}+2 \lambda_{j}^{\frac{1}{2}} R_{0}^{2} k_{0}\right) \\
&+\sum_{j=1}^{n_{1}} C_{26}\left(\left\|\xi_{1 j}\right\|^{2}+\left\|\zeta_{1 j}\right\|^{2}\right) \\
& \leq-\frac{\varepsilon}{8} n_{1}+2 C_{27} \sum_{j=1}^{n_{1}}\left(\lambda_{j}^{-1}+\lambda_{j}^{-1}\right) . \\
& \frac{1}{n_{1}} \sum_{j=1}^{n_{1}}\left(\lambda_{j}^{-1}+\lambda_{j}^{-1}\right) \leq \frac{\varepsilon}{16 C_{27}}, \text { then } \\
& q_{n_{1}}(s)=\liminf _{t \rightarrow \infty} \operatorname{supsup}_{\tau \in R} \sup _{\phi \in E_{0}} \frac{1}{\varphi(\tau) \in \mathrm{A}} \int_{\tau}^{\tau+1} p_{n_{1}}(s) \mathrm{d} s \\
& \leq-2 n_{1} C_{27}\left(\frac{\varepsilon}{16 C_{27}}-\frac{1}{n_{1}} \sum_{j=1}^{n_{1}}\left(\lambda_{j}^{-1}+\lambda_{j}^{-1}\right)\right)<0 .
\end{aligned}
$$

Proof finish.

\section{References}

[1] Lin, G.G. and Zhang, M. (2017) The Global Attractors for a Class of Nonlinear Coupled Kirchhoff-Type Equations. European Journal of Mathematics and Computer Science, 4.

[2] Wu, J.Z. and Lin, G.G. (2009) The Global Attractor and Its Dimensions Estimation of Bossinesq Equation with Damping Term. Journal of Yunnan University, 31, 335340.

[3] Fan, X.M. and Zhou, S.F. (2004) Kernel Sections for Non-Autonomous Strongly Damped Wave Equations of Non-Degenerate Krichhoff-Type. Applied Mathematics and Computation, 158, 253-266. https://doi.org/10.1016/j.amc.2003.08.147

[4] Lin, G.G. and Gao, Y.L. (2017) The Global and Exponential Attractors for the Higher-Order Kirchhoff-Type Equation with Strong Linear Damping. Journal of Mathematics Research, 9.

[5] Teman, R. (1988) Infinite-Dimensional Dynamical Systems in Mechanics and Physics. Applied Mathematical Sciences, 68, Springer-Verlag, New York. https://doi.org/10.1007/978-1-4684-0313-8 